\title{
Serum Potassium Is Associated with Cognitive Decline in Patients with Lewy Body Dementia
}

\author{
Article type: Research Article
}

Authors: Giil, Lasse Melvaer (https://content.iospress.com:443/search?q=author\%3A\%28\%22Giil, Lasse Melvaer\%22\%29) ; ; ; - I Solvang, Stein-Erik Hafstad (https://content.iospress.com:443/search? g=author\%3A\%28\%22Solvang, Stein-Erik Hafstad\%22\%29) ; ; , I Giil, Malin Melvaer (https://content.iospress.com:443/search?q=author\%3A\%28\%22Giil, Malin Melvaer\%22\%29). $\mid$ | Hellton, Kristoffer H. (https://content.iospress.com:443/search?q=author\%3A\%28\%22Hellton, Kristoffer H.\%22\%29). I I Skogseth, Ragnhild Eide (https://content.iospress.com:443/search?q=author\%3A\%28\%22Skogseth, Ragnhild Eide\%22\%29) Vik-Mo, Audun Osland (https://content.iospress.com:443/search?a=author\%3A\%28\%22Vik-Mo, Audun Osland \%22\%29) , ; | | Hortobágyi, Tibor (https://content.iospress.com:443/search?q=author\%3A\%28\%22Hortobágyi, Tibor\%22\%29) ${ }^{\text {g }}$ h | Aarsland, Dag_(https://content.iospress.com:443/search?q=author\%3A\%28\%22Aarsland, Dag\%22\%29) $;$ : $\underline{\text { h }}$ | Nordrehaug, Jan Erik (https://content.iospress.com:443/search?q=author\%3A\%28\%22Nordrehaug, Jan Erik\%22\%29) ; ,

Affiliations: [a] Department of Internal Medicine, Haraldsplass Deaconess Hospital, Bergen, Norway | [b] Institute of Clinical Sciences, University of Bergen, Norway | [c] Semmelweis University, Budapest, Hungary | [d] Norwegian Computing Center, Oslo, Norway | [e] Institute of Clinical Medicine, University of Bergen, Norway I [f] Center for Age-Related Diseases (SESAM), Stavanger University Hospital, Norway | [g] MTA-DE Cerebrovascular and Neurodegenerative Research Group, University of Debrecen, Debrecen, Hungary I [h] Department of Old Age Psychiatry, Institute of Psychiatry, Psychology and Neuroscience, Kings College, UK

Correspondence: $\left[{ }^{*}\right]$ Correspondence to: Lasse Melvaer Giil, MD, Department of Internal Medicine, Haraldsplass Deaconess Hospital, Bergen, Norway. E-mail: lassegiil@gmail.com (mailto:lassegiil@gmail.com).

\begin{abstract}
Background:Epidemiological studies link serum potassium $(\mathrm{K}+)$ to cognitive performance, but whether cognitive prognosis in dementia is related to $\mathrm{K}+$ levels is unknown. Objective:To determine if $\mathrm{K}+$ levels predict cognitive prognosis in dementia and if this varies according to diagnosis or neuropathological findings. Methods:This longitudinal cohort study recruited 183 patients with mild Alzheimer's disease or Lewy body dementia (LBD). Serum K+ and eGFR were measured at baseline and medications which could affect K+ registered. The Mini-Mental State Examination (MMSE) was measured annually over 5 years, and mortality registered. Association between $\mathrm{K}+$ and $\sqrt{ }(30-\mathrm{MMSE})$ was estimated overall, and according to diagnosis (joint model). Associations between MMSE-decline and $\mathrm{K}+$ were assessed in two subgroups with neuropathological examination (linear regression) or repeated measurements of $\mathrm{K}+$ over 3 years (mixed model). Results:Serum $\mathrm{K}+$ at baseline was associated with more errors on MMSE over time (Estimate $0.18, p=0.003)$, more so in LBD $(p=0.048)$. The overall association and LBD interaction were only significant in the 122 patients not using $\mathrm{K}+$ relevant medication. Repeated $\mathrm{K}+$ measures indicated that the association with MMSE errors over time was due to a between-person effect $(p<0.05, n=57)$. The association between the annual MMSE decline was stronger in patients with autopsy confirmed LBD and more $\alpha$-synuclein pathology (all: $p<0.05, n=41$ ). Conclusion:Higher serum $K+$ predicts poorer cognitive prognosis in demented patients not using medications which affect $\mathrm{K}+$, likely a between-person effect seen mainly in LBD.
\end{abstract}

Keywords: $\alpha$-synuclein, Alzheimer's disease, cognitive decline, kalium, Lewy body dementia, Mini-Mental State Examination, MMSE-decline, potassium, prognosis

DOI: 10.3233/JAD-181131

Journal: Journal of Alzheimer's Disease (https://content.iospress.com:443/journals/journal-of-alzheimers-disease), vol. 68, no. 1, pp. 239-253, 2019

Accepted 18 December 2018 | Published: 12 March 2019 